\title{
A polynomial realization of the Hopf algebra of uniform block permutations.
}

\author{
Rémi Maurice ${ }^{1}$ \\ ${ }^{1}$ Institut Gaspard Monge, Université Paris-Est Marne-la-Vallée, 5 Boulevard Descartes, Champs-sur-Marne, 77454 \\ Marne-la-Vallée cedex 2, France
}

\begin{abstract}
We investigate the combinatorial Hopf algebra based on uniform block permutations and we realize this algebra in terms of noncommutative polynomials in infinitely many bi-letters.

Résumé. Nous étudions l'algèbre de Hopf combinatoire dont les bases sont indexées par les permutations de blocs uniformes et nous réalisons cette algèbre en termes de polynômes non-commutatifs en une infinité de bi-lettres.
\end{abstract}

combinatorial Hopf algebras, uniform block permutations

\section{Introduction.}

For several years, Hopf algebras based on combinatorial objects have been thoroughly investigated. Examples include the Malvenuto-Reutenauer Hopf algebra FQSym whose bases are indexed by permutations [7, 4, 2], the Loday-Ronco Hopf algebra PBT whose bases are indexed by planar binary trees [6], the Hopf algebra of free symmetric functions FSym whose bases are indexed by standard Young tableaux [11].

The product and the coproduct, which define the structure of a combinatorial Hopf algebra, are, in general, rules of composition and decomposition given by combinatorial algorithms such as the shuffle and the deconcatenation of permutations, as in FQSym [4], of packed words, as in WQSym [9] or of parking functions, as in PQSym [10]. These can also be given by the disjoint union and the admissible cuts of rooted trees, as in the Connes-Kreimer Hopf algebra [3], or by concatenation and subgraphs/contractions of graphs [8].

Computing with these structures can be difficult, and polynomial realization can bring up important simplifications.

We can see a polynomial realization as the image by an injective Hopf algebra morphism from a combinatorial Hopf algebra to an algebra of polynomials (commutative or not).

The idea is to encode the combinatorial objects by polynomials, in such a way that the product of the combinatorial Hopf algebra become the ordinary product of polynomials, and that the coproduct be given by the disjoint union of alphabets, endowed with some extra structure such as an order relation. This can be done, e.g., for FQSym [4], WQSym [9], PQSym [10] and Connes-Kreimer [5].

In this paper, we realize the Hopf algebra UBP of uniform block permutations introduced by Aguiar and Orellana [1]. We begin by recalling the preliminary notions of uniform block permutations in Section 2. We recall, in Section 3, the Hopf algebra structure of uniform block permutations, we translate this 
structure via the decomposition and we describe the dual structure. Using polynomial realizations of Hopf algebras based on permutations and set partitions, we realize this algebra in Section 4 We finish by finding the Hopf algebra WQSym as a quotient of UBP and the dual as a subalgebra of UBP*.

\section{Acknowledgements}

The author would like to thank Jean-Yves Thibon for his advice and reading of this paper.

\section{Definitions and decompositions.}

Definition 1 A uniform block permutation of size $n$ is a bijection between two set partitions of $\{1, \cdots, n\}$ that maps each block to a block of the same cardinality.

Notation 1 Let $\mathcal{A}=\left\{A_{1}, A_{2}, \cdots, A_{k}\right\}$ and $\mathcal{A}^{\prime}=\left\{A_{1}^{\prime}, A_{2}^{\prime}, \cdots, A_{k}^{\prime}\right\}$ be two set partitions of the same size. We denote the uniform block permutation $f: \mathcal{A} \longrightarrow \mathcal{A}^{\prime}$ for which the image of $A_{i}$ is $A_{i}^{\prime}$ by

$$
\left(\begin{array}{c|c|c|c}
A_{1} & A_{2} & \cdots & A_{k} \\
A_{1}^{\prime} & A_{2}^{\prime} & \cdots & A_{k}^{\prime}
\end{array}\right)
$$

Example 1 Here are all uniform block permutations of size 1 and 2:

$$
\begin{aligned}
& n=1 \\
& n=2
\end{aligned} \quad\left(\begin{array}{l}
1 \\
1
\end{array}\right) .
$$

In the case where the set partitions consist of singletons (i.e., $\mathcal{A}=\{\{1\}, \cdots,\{n\}\}$ ), we find the permutations of size $n$.

Definition 2 Let $f$ and $g$ be two uniform block permutations. The concatenation of $f$ and $g$, denoted by $f \times g$, is the uniform block permutation obtained by adding the size of $f$ to all entries of $g$ and concatenating the result to $f$.

Example 2

$$
\left(\begin{array}{l|l}
13 & 2 \\
23 & 1
\end{array}\right) \times\left(\begin{array}{l|l|l|l}
13 & 2 & 46 & 5 \\
46 & 2 & 15 & 3
\end{array}\right)=\left(\begin{array}{l|l|l|l|l|l}
13 & 2 & 46 & 5 & 79 & 8 \\
23 & 1 & 79 & 5 & 48 & 6
\end{array}\right) .
$$

Definition 3 Let $f: \mathcal{A} \longrightarrow \mathcal{A}^{\prime}$ and $g: \mathcal{B} \longrightarrow \mathcal{B}^{\prime}$ be two uniform block permutations of the same size $n$. The composition $g \circ f: \mathcal{C} \longrightarrow \mathcal{C}^{\prime}$ of $f$ and $g$ is defined by the following process. The blocks $C$ of the set partition $\mathcal{C}$ are the subsets of $\{1, \cdots, n\}$ which are minimal for the two properties

1. $C$ is a union of blocks of $\mathcal{A}$

2. $f(C)$ is a union of blocks $B_{i}$ of $\mathcal{B}$.

The image of $C$ is the union of images $g\left(B_{i}\right)$.

Note that if $f$ is a permutation, then the set partition $\mathcal{C}^{\prime}$ is $\mathcal{B}^{\prime}$, and if $g$ is a permutation, then the set partition $\mathcal{C}$ is $\mathcal{A}$. 


\section{Example 3}

$$
\begin{aligned}
& \left(\begin{array}{l|l}
125 & 346 \\
236 & 145
\end{array}\right) \circ\left(\begin{array}{l|l|l|l|l|l}
1 & 2 & 3 & 4 & 5 & 6 \\
5 & 4 & 2 & 6 & 3 & 1
\end{array}\right)=\left(\begin{array}{ll|l}
136 & 245 \\
236 & 145
\end{array}\right), \\
& \left(\begin{array}{l|l|l|l|l}
15 & 2 & 3 & 4 & 6 \\
46 & 2 & 1 & 5 & 3
\end{array}\right) \circ\left(\begin{array}{l|l|l|l}
14 & 2 & 36 & 5 \\
23 & 1 & 45 & 6
\end{array}\right)=\left(\begin{array}{l|l|l}
14 & 236 & 5 \\
12 & 456 & 3
\end{array}\right) .
\end{aligned}
$$

The data of a permutation and a set partition is enough to describe a uniform block permutation. Given a uniform block permutation $f: \mathcal{A} \longrightarrow \mathcal{A}^{\prime}$ of size $n$, there is a permutation $\sigma$ in $\mathfrak{S}_{n}$ such that

$$
\mathcal{A}^{\prime}=\bigcup_{H \in \mathcal{A}}\left\{\bigcup_{x \in H}\{\sigma(x)\}\right\} \text {. }
$$

Conversely, a set partition of the set $\{1, \cdots, n\}$ and a permutation of $\mathfrak{S}_{n}$ determine a uniform block permutation of size $n$. Any uniform block permutation can thus decompose (non-uniquely) as

$$
f=\sigma \circ I d_{\mathcal{A}}=I d_{\mathcal{A}^{\prime}} \circ \sigma .
$$

The first equality expresses the fact that one can group the images $\sigma_{i}$ whose indices are in the same block in $\mathcal{A}$, the second expresses the fact that one can group the images $\sigma_{i}$ whose values are in the same block in $\mathcal{A}^{\prime}$.

\section{Algebraic structures.}

In [1], Aguiar and Orellana introduced a Hopf algebra whose basis is the set of uniform block permutations. It will be denoted by UBP, standing for Uniform Block Permutations. Let us recall the convolution of two permutations. Let $\tau$ and $\tau^{\prime}$ be two permutations:

$$
\tau * \tau^{\prime}=\sum_{\begin{array}{c}
\sigma=u \cdot v \\
\operatorname{std}(u)=\tau \\
\operatorname{std}(v)=\tau^{\prime}
\end{array}} \sigma
$$

where the standardization is a process that associates a permutation with a word. The standardized of $w$ is defined as the permutation having the same inversions as $w$. For example:

$$
\begin{gathered}
\operatorname{std}\left(a_{2} a_{3} a_{2} a_{1} a_{6} a_{1} a_{4}\right)=3541726, \\
12 * 21=1243+1342+1432+2341+2431+3421 .
\end{gathered}
$$

The product and the coproduct are defined as follows.

Definition 4 Let $f$ and $g$ be two uniform block permutations of respective sizes $m$ and $n$. The convolution, denoted by $f * g$, is the sum of all uniform block permutations of the form

$$
\left(\begin{array}{c|c|c|c}
1 & 2 & \cdots & m+n \\
\sigma_{1} & \sigma_{2} & \cdots & \sigma_{m+n}
\end{array}\right) \circ(f \times g) .
$$

where

$$
\operatorname{std}\left(\sigma_{1} \cdots \sigma_{m}\right)=12 \cdots m \text { and } \operatorname{std}\left(\sigma_{m+1} \cdots \sigma_{m+n}\right)=12 \cdots n
$$

(i.e., $\sigma$ appears in $12 \cdots m * 12 \cdots n$ ). 


\section{Example 4}

$$
\begin{aligned}
\left(\begin{array}{l}
1 \\
1
\end{array}\right) *\left(\begin{array}{l}
12 \\
12
\end{array}\right) & =\left[\left(\begin{array}{l|l|l}
1 & 2 & 3 \\
1 & 2 & 3
\end{array}\right)+\left(\begin{array}{l|l|l}
1 & 2 & 3 \\
2 & 1 & 3
\end{array}\right)+\left(\begin{array}{l|l|l}
1 & 2 & 3 \\
3 & 1 & 2
\end{array}\right)\right] \circ\left(\begin{array}{l|l}
1 & 23 \\
1 & 23
\end{array}\right) \\
& =\left(\begin{array}{l|l}
1 & 23 \\
1 & 23
\end{array}\right)+\left(\begin{array}{l|l}
1 & 23 \\
2 & 13
\end{array}\right)+\left(\begin{array}{l|l}
1 & 23 \\
3 & 12
\end{array}\right) .
\end{aligned}
$$

We denote by $\operatorname{std}(\mathcal{E})$ the standardization of a collection of disjoint sets of integers obtained by numbering each integer $a$ in $\mathcal{E}$ by one plus the number of integers in $\mathcal{E}$ smaller than $a$.

\section{Example 5}

$$
\operatorname{std}(\{\{1,6\},\{9,13\},\{3,5,12\}\})=\{\{1,4\},\{5,7\},\{2,3,6\}\} .
$$

We set

$$
\mathcal{C}_{\mid\{1, \cdots, i\}}=\{H \in \mathcal{C} ; H \subset\{1,2, \cdots, i\}\}, \mathcal{C}_{\mid\{i+1, \cdots, n\}}=\operatorname{std}\left(\mathcal{C}-\mathcal{C}_{\mid\{1, \cdots, i\}}\right)
$$

and let $I(\mathcal{C})$ be the set of integers $i$ such that

$$
\{1,2, \cdots, n\}=\mathcal{C}_{\{1, \cdots, i\}} \times \mathcal{C}_{\mid\{i+1, \cdots, n\}}
$$

for any set partition $\mathcal{C}$.

Example 6 Let $\mathcal{C}=\{\{1,3\},\{2\},\{4,6,7\},\{5\}\}$ be a set partition:

$$
\begin{aligned}
\mathcal{C}_{\{1, \cdots, 2\}} & =\{\{2\}\} & \mathcal{C}_{\{1, \cdots, 3\}} & =\{\{1,3\},\{2\}\} \\
\mathcal{C}_{\{4, \cdots, 7\}} & =\{\{1,3,4\},\{2\}\} & I(\mathcal{C}) & =\{0,3,7\}
\end{aligned}
$$

Definition 5 Let $f: \mathcal{A} \longrightarrow \mathcal{A}^{\prime}$ be a uniform block permutation of size n. By Lemma (2.2) in [1], $i$ is in $I\left(\mathcal{A}^{\prime}\right)$ if and only if there exists a unique permutation $\sigma$ in $\mathfrak{S}_{n}$ appearing in the convolution product $12 \cdots i * 12 \cdots n-i$ and unique uniform block permutations $f_{(i)}$ of size $i$ and $f_{(n-i)}^{\prime}$ of size $n-i$ such that

$$
f=\left(f_{(i)} \times f_{(n-i)}^{\prime}\right) \circ\left(\begin{array}{c|c|c|c}
1 & 2 & \cdots & n \\
\left(\sigma^{-1}\right)_{1} & \left(\sigma^{-1}\right)_{2} & \cdots & \left(\sigma^{-1}\right)_{n}
\end{array}\right) .
$$

The coproduct is then

$$
\Delta(f):=\sum_{i \in I\left(\mathcal{A}^{\prime}\right)} f_{(i)} \otimes f_{(n-i)}^{\prime}
$$

\section{Example 7}

$\Delta\left(\begin{array}{l|l|l}13 & 256 & 4 \\ 15 & 234 & 6\end{array}\right)=(\quad) \otimes\left(\begin{array}{l|l|l}13 & 256 & 4 \\ 15 & 234 & 6\end{array}\right)+\left(\begin{array}{l|l}13 & 245 \\ 15 & 234\end{array}\right) \otimes\left(\begin{array}{l}1 \\ 1\end{array}\right)+\left(\begin{array}{l|l|l}13 & 256 & 4 \\ 15 & 234 & 6\end{array}\right) \otimes(\quad)$

since

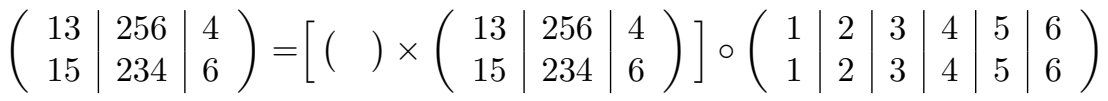

$$
\begin{aligned}
& =\left[\left(\begin{array}{l|l}
13 & 245 \\
15 & 234
\end{array}\right) \times\left(\begin{array}{l}
1 \\
1
\end{array}\right)\right] \circ\left(\begin{array}{l|l|l|l|l|l}
1 & 2 & 3 & 4 & 5 & 6 \\
1 & 2 & 3 & 6 & 4 & 5
\end{array}\right) \\
& \left.=\left[\begin{array}{l|l|l}
13 & 256 & 4 \\
15 & 234 & 6
\end{array}\right) \times(\quad)\right] \circ\left(\begin{array}{l|l|l|l|l|l}
1 & 2 & 3 & 4 & 5 & 6 \\
1 & 2 & 3 & 4 & 5 & 6
\end{array}\right) .
\end{aligned}
$$


One can rewrite this product and coproduct using the decomposition of a uniform block permutation. Given $f: \mathcal{A} \longrightarrow \mathcal{A}^{\prime}$ and $g: \mathcal{B} \longrightarrow \mathcal{B}^{\prime}$ two uniform block permutations, $f=\sigma \circ I d_{\mathcal{A}}=I d_{\mathcal{A}^{\prime}} \circ \sigma$ and $g=\tau \circ I d_{\mathcal{B}}=I d_{\mathcal{B}^{\prime}} \circ \tau$, we have

$$
\begin{aligned}
& f * g=(\sigma * \tau) \circ I d_{\mathcal{A} \times \mathcal{B}} \\
& \Delta(f)=\sum_{i \in I\left(\mathcal{A}^{\prime}\right)} f_{\mid\{1, \cdots, i\}} \otimes f_{\mid\{i+1, \cdots, n\}}
\end{aligned}
$$

where

$$
\begin{aligned}
f_{\mid\{1, \cdots, i\}} & =I d_{\mathcal{A}_{\mid\{1, \cdots, i\}}^{\prime}} \circ \sigma_{\mid\{1, \cdots, i\}} \\
f_{\mid\{i+1, \cdots, n\}} & =I d_{\mathcal{A}_{\mid\{i+1, \cdots, n\}}^{\prime}} \circ \operatorname{std}\left(\sigma_{\mid\{i+1, \cdots, n\}}\right) .
\end{aligned}
$$

\section{Example 8}

$$
\begin{aligned}
\left(\begin{array}{c}
1 \\
1
\end{array}\right) *\left(\begin{array}{c}
12 \\
12
\end{array}\right) & =1 \circ I d_{\{\{1\}\}} * 12 \circ I d_{\{\{1,2\}\}} \\
& =(1 * 12) \circ I d_{\{\{1\},\{2,3\}\}} \\
& =(123+213+312) \circ I d_{\{\{1\},\{2,3\}\}} \\
& =\left(\begin{array}{c|c}
1 & 23 \\
1 & 23
\end{array}\right)+\left(\begin{array}{l|l}
1 & 23 \\
2 & 13
\end{array}\right)+\left(\begin{array}{l|l}
1 & 23 \\
3 & 12
\end{array}\right) .
\end{aligned}
$$

Example 9 We set $\mathcal{A}=\{\{1,5\},\{2,3,4\},\{6\}\}$.

$$
\begin{aligned}
\Delta\left(\begin{array}{l|l|l}
13 & 256 & 4 \\
15 & 234 & 6
\end{array}\right) & =\Delta\left(I d_{\mathcal{A}} \circ 125634\right) \\
& =\varepsilon \otimes I d_{\mathcal{A}} \circ 125634+I d_{\{\{1,5\},\{2,3,4\}\}} \circ 12534 \otimes I d_{\{\{1\}\}} \circ 1+I d_{\mathcal{A}} \circ 125634 \otimes \varepsilon \\
& =(\quad) \otimes\left(\begin{array}{l|l|l|l}
13 & 256 & 4 \\
15 & 234 & 6
\end{array}\right)+\left(\begin{array}{c|c|c|c}
13 & 245 \\
15 & 234
\end{array}\right) \otimes\left(\begin{array}{l}
1 \\
1
\end{array}\right)+\left(\begin{array}{l|l|l}
13 & 256 & 4 \\
15 & 234 & 6
\end{array}\right) \otimes(\quad) .
\end{aligned}
$$

It is proved in [1] that UBP is a self-dual Hopf algebra. We can describe the dual structure. Let us first define another product and coproduct of uniform block permutations. Let $f: \mathcal{A} \longrightarrow \mathcal{A}^{\prime}$ and $g: \mathcal{B} \longrightarrow \mathcal{B}^{\prime}$ be two uniform block permutations, $f=\sigma \circ I d_{\mathcal{A}}=I d_{\mathcal{A}^{\prime}} \circ \sigma$ and $g=\tau \circ I d_{\mathcal{B}}=I d_{\mathcal{B}^{\prime}} \circ \tau$ :

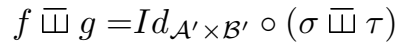

$$
\begin{aligned}
& \Delta_{1}(f)=\sum_{i \in I(\mathcal{A})} f_{1} \cdots f_{i} \otimes f_{i+1} \cdots f_{n}
\end{aligned}
$$

where

$$
\begin{aligned}
f_{1} \cdots f_{i} & =\operatorname{std}\left(\sigma_{1} \cdots \sigma_{i}\right) \circ I d_{\mathcal{A}_{\mid\{1, \cdots, i\}}} \\
f_{i+1} \cdots f_{n} & =\operatorname{std}\left(\sigma_{i+1} \cdots \sigma_{n}\right) \circ I d_{\mathcal{A}_{\mid\{i+1, \cdots, n\}}}
\end{aligned}
$$




\section{Example 10}

$$
\begin{aligned}
& \left(\begin{array}{c}
1 \\
1
\end{array}\right) \bar{\Psi}\left(\begin{array}{c}
12 \\
12
\end{array}\right)=I d_{\{\{1\},\{2,3\}\}} \circ(1 \bar{\Psi} 12) \\
& =I d_{\{\{1\},\{2,3\}\}} \circ(123+213+231) \\
& =\left(\begin{array}{l|l}
1 & 23 \\
1 & 23
\end{array}\right)+\left(\begin{array}{l|l}
2 & 13 \\
1 & 23
\end{array}\right)+\left(\begin{array}{l|l}
3 & 12 \\
1 & 23
\end{array}\right) . \\
& \Delta_{1}\left(\begin{array}{l|l|l}
13 & 256 & 4 \\
15 & 234 & 6
\end{array}\right)=\Delta_{1}\left(125634 \circ I_{\{\{1,3\},\{2,5,6\},\{4\}\}}\right) \\
& =\varepsilon \otimes 125634 \circ I d_{\{\{1,3\},\{2,5,6\},\{4\}\}}+125634 \circ I d_{\{\{1,3\},\{2,5,6\},\{4\}} \otimes \varepsilon
\end{aligned}
$$

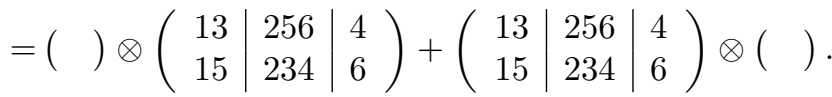

The two structures of Hopf algebra are dual to each other for the scalar product

$$
\langle f, g\rangle=\delta_{f, g^{-1}} .
$$

In other words, we have:

$$
\begin{aligned}
\langle\Delta f, g \otimes h\rangle & =\langle f, g \varpi h\rangle \\
\left\langle\Delta_{1} f, g \otimes h\right\rangle & =\langle f, g * h\rangle .
\end{aligned}
$$

\section{Polynomial realization.}

Given two alphabets $B=\left\{b_{i} ; 0 \leq i\right\}$ and $C=\left\{c_{i} ; 0 \leq i\right\}$, with $C$ totally ordered, we consider an alphabet $A=\left\{\left(\begin{array}{c}b_{i} \\ c_{j}\end{array}\right) ; 0 \leq i, j\right\}$ of bi-letters endowed with two relations:

- for all $i$ and $k,\left(\begin{array}{c}b_{i} \\ c_{j}\end{array}\right) \preceq\left(\begin{array}{c}b_{k} \\ c_{l}\end{array}\right)$ if and only if $c_{j} \leq c_{l}$,

- for all $j$ and $l,\left(\begin{array}{c}b_{i} \\ c_{j}\end{array}\right) \equiv\left(\begin{array}{l}b_{k} \\ c_{l}\end{array}\right)$ if and only if $b_{i}=b_{k}$.

The product of two biwords is the concatenation

$$
\left(\begin{array}{l}
u_{1} \\
v_{1}
\end{array}\right)\left(\begin{array}{l}
u_{2} \\
v_{2}
\end{array}\right)=\left(\begin{array}{l}
u_{1} u_{2} \\
v_{1} v_{2}
\end{array}\right)
$$

We extend the definition of the standardization to biwords. Let $w=\left(\begin{array}{l}u \\ v\end{array}\right)$ be a biword. The standardized of $w$ is the permutation $\operatorname{std}(v)$. 


\section{Example 11}

$$
\operatorname{std}\left[\left(\begin{array}{l}
b_{1} b_{1} b_{3} b_{1} b_{2} \\
c_{2} c_{3} c_{2} c_{1} c_{6}
\end{array}\right)\right]=24315 .
$$

Let $f: \mathcal{A} \longrightarrow \mathcal{A}^{\prime}$ be a uniform block permutation. Let $\xi_{f}$ be the minimum permutation (for the lexicographic order) such that $f=\xi_{f} \circ I d_{\mathcal{A}}$. We say that a biword $w$ is $f$-compatible if

$$
-\operatorname{std}(w)=\xi_{f}
$$

and

- if $i$ and $j$ are in the same block in $\mathcal{A}$, then the bi-letters $w_{i}$ and $w_{j}$ satisfy $w_{i} \equiv w_{j}$.

We set:

$$
\mathbf{G}_{f}(A)=\sum_{w ; w \text { is } f-\text { compatible }} w .
$$

Example 12 Let $f=\left(\begin{array}{l|l|l}13 & 256 & 4 \\ 15 & 234 & 6\end{array}\right)$ be a uniform block permutation. The permutation $\sigma=125634$ is the minimum permutation associated with $f$ and we have

$$
\mathbf{G}_{f}(A)=\sum_{\substack{i, j, k \\
\operatorname{std}\left(c_{1} c_{2} \cdots c_{6}\right)=\sigma}}\left(\begin{array}{c}
b_{i} b_{j} b_{i} b_{k} b_{j} b_{j} \\
c_{1} c_{2} c_{3} c_{4} c_{5} c_{6}
\end{array}\right) .
$$

Theorem 1 The polynomials $\mathbf{G}_{f}(A)$ provide a realization of the Hopf algebra of uniform block permutations. That is to say

- $\mathbf{G}_{f}(A) \mathbf{G}_{g}(A)=\sum_{h \in f * g} \mathbf{G}_{h}(A)$

- Let $A^{\prime}$ be an alphabet isomorphic to the alphabet $A$ such that, for any bi-letter $w^{\prime}$ of $A^{\prime}$ and for any bi-letter $w$ of $A$, we have

$$
w \preceq w^{\prime} .
$$

Define $A+A^{\prime}$ as the disjoint union of $A$ and $A^{\prime}$ endowed with the relations $\preceq$ and $\equiv$. If we allow the bi-letters of $A$ and $A^{\prime}$ to commute and identify $P(A) Q\left(A^{\prime}\right)$ with $P \otimes Q$, we have:

$$
\mathbf{G}_{f}\left(A+A^{\prime}\right)=\Delta\left(\mathbf{G}_{f}\right) \text {. }
$$

\section{Example 13}

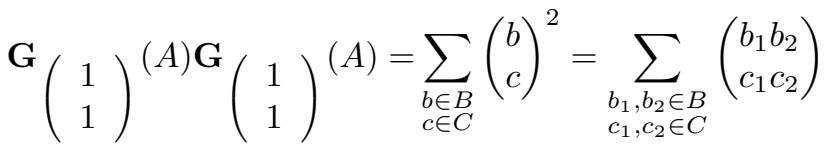

$$
\begin{aligned}
& =\sum_{\substack{b_{1}, b_{2} \in B \\
c_{1}, c_{2} \in C ; c_{1} \leq c_{2}}}\left(\begin{array}{l}
b_{1} b_{2} \\
c_{1} c_{2}
\end{array}\right)+\sum_{\substack{b_{1}, b_{2} \in B \\
c_{1}, c_{2} \in C ; c_{1}>c_{2}}}\left(\begin{array}{l}
b_{1} b_{2} \\
c_{1} c_{2}
\end{array}\right) \\
& =\mathbf{G}\left(\begin{array}{l|l}
1 & 2 \\
1 & 2
\end{array}\right)^{(A)+\mathbf{G}}\left(\begin{array}{l|l}
1 & 2 \\
2 & 1
\end{array}\right)^{(A) .}
\end{aligned}
$$




$$
\begin{aligned}
\mathbf{G}\left(\begin{array}{l}
12 \\
12
\end{array}\right)^{(A) \mathbf{G}}\left(\begin{array}{l}
1 \\
1
\end{array}\right)= & \sum_{\substack{b \in B \\
\operatorname{std}(v)=12}}\left(\begin{array}{c}
b b \\
v
\end{array}\right) \sum_{\substack{b \in B \\
c \in C}}\left(\begin{array}{l}
b \\
c
\end{array}\right) \\
= & \sum_{\substack{i_{1}, i_{2} \\
\operatorname{std}\left(v_{1}=12 \\
\operatorname{std}\left(v_{2}\right)=1\right.}}\left(\begin{array}{c}
b_{i_{1}} b_{i_{1}} b_{i_{2}} \\
v_{1} v_{2}
\end{array}\right) \\
= & \sum_{\substack{i_{1}, i_{2} \\
c_{1} \leq c_{2} \leq c_{3}}}\left(\begin{array}{c}
b_{i_{1}} b_{i_{1}} b_{i_{2}} \\
c_{1} c_{2} c_{3}
\end{array}\right)+\sum_{\begin{array}{c}
i_{1}, i_{2} \\
c_{1} \leq c_{3}<c_{2}
\end{array}}\left(\begin{array}{c}
b_{i_{1}} b_{i_{1}} b_{i_{2}} \\
c_{1} c_{2} c_{3}
\end{array}\right)+\sum_{\substack{i_{1}, i_{2} \\
c_{3}<c_{1} \leq c_{2}}}\left(\begin{array}{c}
b_{i_{1}} b_{i_{1}} b_{i_{2}} \\
c_{1} c_{2} c_{3}
\end{array}\right) \\
= & \mathbf{G}\left(\begin{array}{c|c}
12 & 3 \\
12 & 3
\end{array}\right)+\mathbf{G}\left(\begin{array}{c|c}
12 & 3 \\
13 & 2
\end{array}\right)+\mathbf{G}\left(\begin{array}{c|c}
12 & 3 \\
23 & 1
\end{array}\right) .
\end{aligned}
$$

Example 14 We set $\tau=2431$.

$$
\begin{aligned}
& \mathbf{G}\left(\begin{array}{l|l}
13 & 24 \\
13 & 24
\end{array}\right)\left(A+A^{\prime}\right)=\sum_{\substack{i, j \\
c_{1} \leq c_{2} \leq c_{3} \leq c_{4}}}\left(\begin{array}{c}
b_{i} b_{j} b_{i} b_{j} \\
c_{1} c_{2} c_{3} c_{4}
\end{array}\right)+\sum_{\substack{i, j \\
c_{1}^{\prime} \leq c_{2}^{\prime} \leq c_{3}^{\prime} \leq c_{4}^{\prime}}}\left(\begin{array}{c}
b_{i}^{\prime} b_{j}^{\prime} b_{i}^{\prime} b_{j}^{\prime} \\
c_{1}^{\prime} c_{2}^{\prime} c_{3}^{\prime} c_{4}^{\prime}
\end{array}\right) \\
& =\mathbf{G}\left(\begin{array}{l|l}
13 & 24 \\
13 & 24
\end{array}\right)^{(A)+\mathbf{G}}\left(\begin{array}{l|l}
13 & 24 \\
13 & 24
\end{array}\right)^{\left(A^{\prime}\right) .}
\end{aligned}
$$

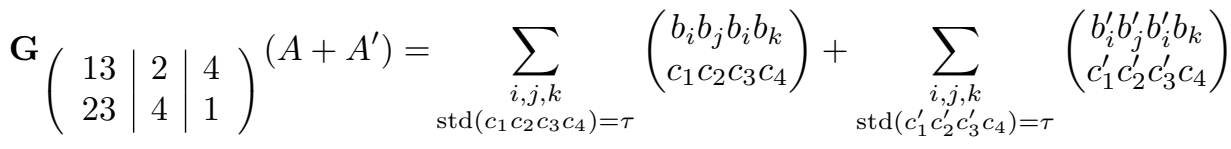

$$
\begin{aligned}
& +\sum_{\substack{i, j, k \\
\operatorname{std}\left(c_{1} c_{2}^{\prime} c_{3} c_{4}\right)=\tau}}\left(\begin{array}{c}
b_{i} b_{j}^{\prime} b_{i} b_{k} \\
c_{1} c_{2}^{\prime} c_{3} c_{4}
\end{array}\right)+\sum_{\substack{i, j, k \\
\operatorname{std}\left(c_{1}^{\prime} c_{2}^{\prime} c_{3}^{\prime} c_{4}^{\prime}\right)=\tau}}\left(\begin{array}{c}
b_{i}^{\prime} b_{j}^{\prime} b_{i}^{\prime} b_{k}^{\prime} \\
c_{1}^{\prime} c_{2}^{\prime} c_{3}^{\prime} c_{4}^{\prime}
\end{array}\right) \\
& =\mathbf{G}\left(\begin{array}{l|l|l}
13 & 2 & 4 \\
23 & 4 & 1
\end{array}\right)^{(A)+\mathbf{G}}\left(\begin{array}{l}
1 \\
1
\end{array}\right)^{(A) \mathbf{G}}\left(\begin{array}{l|l|l}
13 & 2 \\
12 & 3
\end{array}\right)^{\left(A^{\prime}\right)}
\end{aligned}
$$



\section{Packed words.}

We recall the definition of a packed word and the structure of Hopf algebra given in [9]. The packed word $\operatorname{pack}(w)$ associated with a word $w \in A^{*}$ is the word $u$ obtained by the following process. If $b_{1}<b_{2}<\cdots<b_{r}$ are the letters occuring in $w, u$ is the image of $w$ by the homomorphism $b_{i} \longrightarrow a_{i}$. A word $w$ is said to be packed if $\operatorname{pack}(w)$ is $w$. 


\subsection{WQSym*.}

The Hopf algebra WQSym* is the $\mathbb{K}$-vector space generated by packed words endowed with the shifted shuffle product and the coproduct given by, using notations in [5]:

$$
\begin{aligned}
u \bar{\varpi} v & =u \amalg v[\max (u)], \\
\Delta(w) & =\sum_{u \cdot v=w} \operatorname{pack}(u) \otimes \operatorname{pack}(v) .
\end{aligned}
$$

Let $\Psi$ be the map that associates the word $w$ of the length $n$ with a permutation $\sigma$ of size $n$ and a set partition $\mathcal{A}$ of $\{1,2, \cdots, n\}$ constructed by the following process. The $i$ th letter $u_{i}$ of $u$ is the minimum of the letters $\sigma_{j}$ whose values $\sigma_{j}$ are in the same block in $\mathcal{A}$ as $\sigma_{i}$. The word $w$ is obtained by packing. The map $\Psi$ induces a linear map from the vector space generated by uniform block permutations to that generated by packed words. We define it by writing uniform block permutations $f$ in the form $I d_{\mathcal{A}^{\prime}} \circ \sigma$ and applying $\Psi$ to $\sigma$ and $\mathcal{A}^{\prime}$. We also call this map $\Psi$.

\section{Example 15}

$$
\Psi\left[\left(\begin{array}{l}
12 \\
12
\end{array}\right)\right]=11 \quad \Psi\left[\left(\begin{array}{l|l}
12 & 3 \\
23 & 1
\end{array}\right)\right]=221 \quad \Psi\left[\left(\begin{array}{l|l}
13 & 24 \\
12 & 34
\end{array}\right)\right]=1212
$$

If we endow the vector spaces with the shifted shuffle product, the map $\Psi$ is an algebra morphism.

Proposition 1 Let $f$ and $g$ be two uniform block permutations.

$$
\Psi(f \bar{\amalg} g)=\Psi(f) \bar{\Psi} \Psi(g) .
$$

\section{Example 16}

$$
\begin{aligned}
\Psi\left[\left(\begin{array}{l|l}
12 & 3 \\
13 & 2
\end{array}\right) \bar{\Psi}\left(\begin{array}{l|l}
1 & 2 \\
1 & 2
\end{array}\right)\right]= & 11234+11324+11342+13124+13142+ \\
& 13412+31124+31142+31412+34112 \\
= & 112 \bar{\Psi} 12 \\
= & \Psi\left[\left(\begin{array}{l|l}
12 & 3 \\
13 & 2
\end{array}\right)\right] \Psi \Psi\left[\left(\begin{array}{l|l}
1 & 2 \\
1 & 2
\end{array}\right)\right] .
\end{aligned}
$$

The map $\Psi$ cannot be a coalgebra morphism as shown in the example

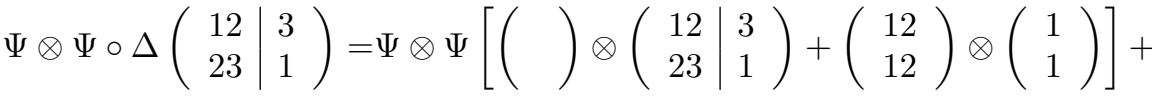

$$
\begin{aligned}
& \Psi \otimes \Psi\left[\left(\begin{array}{l|l}
12 & 3 \\
23 & 1
\end{array}\right) \otimes(\quad)\right] \\
& =\varepsilon \otimes 221+11 \otimes 1+221 \otimes \varepsilon \\
& \neq \varepsilon \otimes 221+1 \otimes 21+11 \otimes 1+221 \otimes \varepsilon \\
& \neq \Delta(221) \text {. }
\end{aligned}
$$


The idea is that the coproduct on packed words deconcatenates the word in all suffixes and prefixes, whereas the coproduct on uniform block permutations preserves the blocks. This suggests to define an another coproduct:

$$
\Delta^{\prime}(w)=\sum_{\substack{u \cdot v=w \\ \operatorname{alph}(u) \cap \operatorname{lph}(v)=\emptyset}} \operatorname{pack}(u) \otimes \operatorname{pack}(v) .
$$

Endowed with the shifted shuffle product $\bar{W}$ and with the coproduct $\Delta^{\prime}$, the vector space generated by packed words is a combinatorial Hopf algebra denoted by WQSym*'.

Proposition 2 The Hopf algebras WQSym* and WQSym*' are isomorphic.

The morphism of Hopf algebras is given by the following process. We define the following relation on packed words of the same length. Let $u$ and $v$ be two packed words, the cover relation is $u \preceq v$ if and only if

- $\operatorname{std}(u)=\operatorname{std}(v)$

- there exists a $i$ such that $u_{k}=\left\{\begin{array}{cl}v_{k} & \text { if } v_{k} \leq i \\ v_{k}-1 & \text { otherwise. }\end{array}\right.$

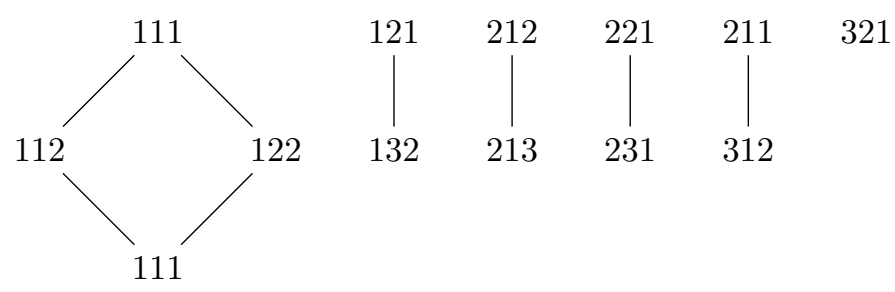

The map $\Gamma$ defined as

$$
\begin{array}{ccc}
\text { WQSym }^{*} & \longrightarrow & \text { WQSym }^{*}, \\
w & \longmapsto & \sum_{u \succeq w} u
\end{array}
$$

is an isomorphism of Hopf algebras. For example, the image of a permutation is a permutation, the image of the word of length $n$ consisting only of 1 is the sum of all the non-decreasing packed words of length $n$.

Example 17

$$
\begin{aligned}
& \Gamma(11 \varpi 1)=\Gamma(112+121+211) \\
& =112+123+121+132+311+312 \\
& =11 \varpi 1+12 \varpi 1 \\
& =\Gamma(11) \bar{\amalg} \Gamma(1) \\
& \Gamma \otimes \Gamma(\Delta(121))=121 \otimes \varepsilon+132 \otimes \varepsilon+12 \otimes 1+1 \otimes 21+\varepsilon \otimes 121+\varepsilon \otimes 132 \\
& =\Delta^{\prime}(121)+\Delta^{\prime}(132) \\
& =\Delta^{\prime}(\Gamma(121)) \text {. }
\end{aligned}
$$

Proposition 3 The map $\Psi$ is a surjective Hopf algebra morphism from UBP* to WQSym*'. 


\subsection{WQSym.}

We denote by WQSym the $\mathbb{K}$-vector space generated by the packed words endowed with the convolution product and the coproduct given by

$$
\begin{aligned}
u *_{W} v & =\sum_{w=u^{\prime} \cdot v^{\prime} \in P W ; \operatorname{pack}\left(u^{\prime}\right)=u, \operatorname{pack}\left(v^{\prime}\right)=v} w, \\
\Delta(u) & =\sum_{0 \leq k \leq \max (u)} u_{\{1, \cdots, k\}} \otimes \operatorname{pack}\left(u_{\mid\{k+1, \cdots, \max (u)\})}\right)
\end{aligned}
$$

The dual WQSym' of WQSym*', is the Hopf algebra endowed with the product

$$
u *_{W}^{\prime} v=\sum_{\begin{array}{c}
w=u^{\prime} \cdot v^{\prime} \in P W \\
\operatorname{pack}\left(u^{\prime}\right)=u \\
\operatorname{pack}\left(v^{\prime}\right)=v \\
\operatorname{alph}(u) \operatorname{alph}(v)=\emptyset
\end{array}} w
$$

for any packed word $u$ and $v$, and the coproduct $\Delta$.

Proposition 4 The Hopf algebra WQSym' and WQSym are isomorphic.

In fact, the map $\Gamma$ induces a dual map. This is

$$
\begin{array}{ccc}
\text { WQSym' } & \longrightarrow & \text { WQSym } \\
\Gamma^{*}: \begin{array}{c}
\text { WQS } \\
w
\end{array} & \longmapsto & \sum_{u \preceq w} u .
\end{array}
$$

For example:

$$
\Gamma^{*}(1212334)=1212334+1212223+1212333+1212222 .
$$

This map is an isomorphism of Hopf algebras.

\section{Example 18}

$$
\begin{aligned}
\Gamma^{*}\left(212 *_{W}^{\prime} 11\right) & =\Gamma^{*}(21233+31322+32311) \\
& =(21233+21222)+(31322+21211)+32311 \\
& =212 * W 11 \\
& =\Gamma^{*}(212) * W \Gamma^{*}(11) \\
\Gamma^{*} \otimes \Gamma^{*} \circ \Delta(312341)= & \Gamma^{*} \otimes \Gamma^{*}(\varepsilon \otimes 312341+11 \otimes 2123+121 \otimes 112+31231 \otimes 1+312341 \otimes \varepsilon) \\
= & \varepsilon \otimes(312341+312331)+11 \otimes(2123+2122)+ \\
& 121 \otimes(112+111)+31231 \otimes 1+(312341+312331) \otimes \varepsilon \\
= & \Delta(312341+312331) \\
= & \Delta\left(\Gamma^{*}(312341)\right)
\end{aligned}
$$


Proposition 5 The map

$$
\begin{array}{cl}
\varphi: \text { WQSym }^{\prime} & \longrightarrow \\
w & \longmapsto \sum_{f ; \Psi(f)=w}^{\text {UBP }} f
\end{array}
$$

is an injective morphism of Hopf algebras.

\section{References}

[1] M. Aguiar and R.C. Orellana. The Hopf algebra of uniform block permutations. J. Alg. Combin., 28 (1):115-138, 2008.

[2] M. Aguiar and F. Sottile. Structure of the Malvenuto-Reutenauer Hopf algebra of permutations. Adv. Math., 191(2):225-275, 2005.

[3] A. Connes and D. Kreimer. Hopf algebras, renormalization and noncommutative geometry. Comm. Math. Phys, pages 203-242, 1998.

[4] G. Duchamp, F. Hivert, and J.-Y. Thibon. Noncommutative symmetric functions VI: Free quasisymmetric functions and related algebras. Internat. J. Alg. Comput., 12:671-717, 2002.

[5] L. Foissy, J.-C. Novelli, and J.-Y. Thibon. Polynomial realizations of some combinatorial Hopf algebras. arXiv:1009.2067v1 [math.CO], 2010.

[6] J.-L. Loday and M.-O. Ronco. Hopf algebra of the planar binary trees. Adv. Math., 139:293-309, 1998.

[7] C. Malvenuto and C. Reutenauer. Duality between quasi-symmetric functions and the Solomon descent algebra. J. alg., 177:967-982, 1995.

[8] D. Manchon. On bialgebras and Hopf algebras of oriented graphs. arXiv:1011.3032v3 [math.CO], 2011.

[9] J.-C. Novelli and J.-Y. Thibon. Polynomial realizations of some trialgebras. FPSAC (San Diego), 2006.

[10] J.-C. Novelli and J.-Y. Thibon. Hopf algebras and dendriform structures arising from parking functions. Fundamenta Mathematicae, 193:189-241, 2007.

[11] S. Poirier and C. Reutenauer. Algèbres de Hopf de tableaux. Ann. Sci. Math. Québec, 19:79-90, 1995. 